\title{
BIOLOGÍA DE Brachmia convolvuli Walsingham (Lepidoptera: Gelechiidae)
}

\author{
Menandro S. Ortiz ${ }^{1}$ \\ Mario E. Cahuana ${ }^{2}$ \\ Verónica E. Rubín de Celis ${ }^{3}$
}

\begin{abstract}
RESUMEN
Con la finalidad de fijar las bases para el conocimiento de la biología de Brachmia convolvuli Walsinghan y ser utilizados con posteridad en investigaciones, así como en los diseños de programas de control, se llevó a cabo el presente trabajo en los Laboratorios de Entomología de la Universidad Nacional Agraria La Molina, bajo condiciones controladas de temperatura $\left(27^{\circ} \mathrm{C} \pm 1^{\circ} \mathrm{C}\right.$ ) y humedad relativa (HR $55 \% \pm 5 \%$ ). Las larvas se criaron en hojas de camote var. Maleña. Los adultos fueron alimentados con solución de miel y agua en proporción de 3:1. Para el estudio de la capacidad de oviposición, fecundidad, fertilidad, períodos de preoviposición y post-oviposición; las parejas se formaron de la siguiente manera: a) de edades iguales, b) la hembra es mayor y c) la hembra es menor. La pupa que origina machos tiene una duración de 10.2 días, mientras que 9.68 días lo es para la hembra. Los períodos de preoviposición, oviposición y post-oviposición son de 5.09, 21.28 y 1.92 días, respectivamente. La fecundidad es de 187.40 huevos de los cuales la mayor parte son ovipuestos durante los 10 primeros días. La fertilidad más alta (96.74\%) se obtiene cuando la pareja tiene la misma edad. La longevidad del adulto es de 28.29 días. Brachmia convolvuli para completar su ciclo biológico necesita 65 días.
\end{abstract}

Palabras claves: Biología de insectos, Brachmia, Gelechiidae, camote.

\section{SUMMARY}

With the objective of stablish the bases for the knowledge of the Brachmia convolvuli Walsingham biology and it's utilization with posterity on researchs, just as the designs of controled programs, the present work has been realized in the Entomology Laboratory of the Universidad Nacional Agraria La Molina, under controlled temperature conditions $\left(27^{\circ} \mathrm{C} \pm\right.$ $1^{\circ} \mathrm{C}$ ) and relative humidity (HR 55\% $\pm 5 \%$ ). The larvae were breed on sweet potatoes leafs (variety Maleña). The adults were feed with a solution of honey and water on proportion 3:1. For capability studies of oviposition, fecundity, fertility, pre-oviposition and post-oviposition, the parteners were conformed as follows: a) same ages, b) the female is the most oldest, c) the females is young. The pupae which origins males last 10,2 days, while the females lasts 9,68 days. The periods of pre-oviposition, oviposition and post-oviposition is of 5,09; 21,28 and 1,92 days, respectively. The fecundity is near to 187,40 eggs, from wich the majority are ovipositioned during the first 10 days. The highest fertility $(96,74 \%)$ is obtaiened when the copuple has the same age. The longevity of the adult is of 28,29 days. Brachmia convolvuli to completes its biological cycle requires 65 days.

Key Words: Insects biology, Brachmia, Gelechiidae, sweet potatoes.

\footnotetext{
${ }^{1}$ Facultad de Ciencias Biológicas, Universidad Ricardo Palma; Avenida Benavides 5440, Lima-Perú; e-mail:mop@infonegocio.net.pe

${ }^{2}$ Universidad Nacional Agraria La Molina, Apartado 456 Lima- Perú.

${ }^{3}$ Instituto de Ciencia y Tecnología, Laboratorio de Genómica y Biología Molecular Evolutiva, Universidad Ricardo Palma, Lima-Perú; e- mail: afidi2001@hotmail.com
} 


\section{INTRODUCCIÓN}

El camote es un cultivo que representa una gran importancia en el país, debido a su alto valor nutritivo, rusticidad, posibilidad de siembra durante todo el año, facilidad de manejo del cultivo, menor inversión que en otros cultivos, que lo hace más económico.

Sin embargo, como en todo cultivo, existen factores que disminuyen el rendimiento por unidad de área. Dentro de éstos se puede mencionar la disponibilidad de agua, plagas y otros factores.

Las especies de insectos plagas infestan a éste cultivo durante todo su desarrollo causando daños de acuerdo a su incidencia, la misma que está determinada por los factores ambientales, presencia o ausencia de enemigos naturales, aplicación de insecticidas y otras causas.

Entre las especies plaga citadas para el país se menciona a Euscepes postfasciatus como la especie de mayor importancia, Empoasca fabalis, Macrosiphum euphorbiae, Microthyris anormalis, Brachmia convolvuli y Ochyrotica fasciata.

La información sobre los aspectos biológicos fundamentales de Brachmia convolvuli no están bien determinados a excepción de las citadas por Wille (1952). En consecuencia, se precisa realizar trabajos de investigación que permitan conocer la biología de esta especie, para que sirvan de base para el desarrollo de futuros programas de manejo de éste cultivo.

\section{ANTECEDENTES}

El camote es un cultivo que tiene gran variabilidad genética, el que viene aprovechándose para seleccionar variedades, tomando en consideración diferentes características de la planta con son el rendimiento, resistencia a plagas y enfermedades, hábitos de crecimiento, así como el valor nutritivo y el color de la pulpa de las raíces a ser utilizadas en la alimentación humana y/o animal.
Dentro de los factores sanitarios en sus diferentes estados de desarrollo, figuran inicialmente los gusanos cortadores, luego una serie de especies de insectos que se alimentan de las hojas y finalmente otros que atacan a las raíces, provocando daños directos o simplemente abriendo puertas de entrada a microorganismos que la descomponen y/o le dan sabores muy desagradables. Estos daños conducen a una reducción de la producción.

Asì, Wille (1952) menciona a Euscepes batatae como una especie que ataca raíces, concediéndole un sabor similar al de carne podrida. Según Cetraro y Ortiz (1982), las especies plaga de mayor importancia son Macrosiphum euphorbiae, Empoasca fabalis y Calycomyza ipomoea, mientras que los que se presentan en poblaciones relativamente pequeñas e infrecuentes son Bemisia sp, tisanópteros, Brachmia convolvuli, Anthicus sp, Ochyrotica fasciata, Diabrotica decolor y Myzus persicae, entre otras especies.

Sin embargo éstos grupos de menor importancia no debe ser ignorado, ya que la importancia podría se mayor, al romperse la ruptura del equilibrio biológico; lo que puede ser causado por el uso creciente e indiscriminado de productos agroquímicos. Por otro lado el incremento de superficies sembradas, aumenta la posibilidad de presencia de plagas. Cabe mencionar que con la creación de variedades de alguna manera podría perderse la resistencia natural que puede haber contra algunas plagas.

En nuestro medio se cuenta con poca información en relación a Brachmia convolvuli. Sarmiento (1983) señala que la larva de Brachmia convolvuli, en su último estadío de desarrollo alcanza una longitud de 8 - $10 \mathrm{~mm}$. García (1984) adiciona que las alas de Brachmia convolvuli plegadas sobre el cuerpo, le otorga un aspecto triangular. Agrega que los huevos son muy pequeños, ovalados y blanco amarillentos. Sobre su comportamiento, Wille (1952) menciona que las larvas de ésta especie atacan no solamente a las variedades moradas 
(«italianas») de hojas delgadas y trilobada, sino también a las de hojas anchas.

\section{MATERIALES Y MÉTODO}

El presente trabajo se realizó en Laboratorios de Entomología de la Universidad Nacional Agraria La Molina, bajo condiciones controladas de temperatura y humedad relativa $\left(27^{\circ} \mathrm{C} \pm 1^{\circ} \mathrm{C}\right.$ y HR 55\% $\pm 5 \%$ ).

Para la biología de Brachmia convolvuli se colectó larvas de un sembrío de camote ubicado en un campo de la Universidad Nacional Agraria La Molina, las que se trasladaron al laboratorio, a fin de iniciar la crianza masal. Obtenidas las pupas, se procedió a determinar el sexo de cada una de ellas, así como tomar las mediciones requeridas.

Una vez que emergieron los adultos, se formaron parejas a las que se les confinó en una unidad de apareo y oviposición. Las parejas fueron dispuestas en tres tratamientos. En el primer tratamiento, la hembra y el macho emergieron el mismo día, en el segundo la hembra fue mayor y en el tercer tratamiento la hembra fue menor que el macho. Se alimentó a los adultos con una solución azucarada en proporción de 3:1. Se registró diariamente el número de huevos puestos y eclosionados, lo que sirvió para determinar la fertilidad.

De estas generaciones de laboratorio, se tomaron 25 huevos, los que se observaron diariamente, tomándose datos sobre los cambios que ocurren durante la incubación. A la eclosión de los huevos se registró la fecha de la ocurrencia e inmediatamente las pequeñas larvas fueron confinadas en unidades de crianza individuales, observándose la mudas que ocurren y en donde se les practicó las mediciones pertinentes.

Las pupas y los adultos fueron conducidos y evaluados similarmente a las que se realizaron en la generación de campo. Se condujo y evaluó dos generaciones de laboratorio; y simultáneamente a cada una de éstas se llevó a cabo crianzas masales, cuya finalidad fue disponer de individuos de edad y sexo requerido cuando fue necesario.

Una vez concluida la parte experimental, se realizó pruebas de Student para determinar el nivel de significancia de la diferencia de promedios para los parámetros de cada uno de los estados inmaduros, así como para los diferentes estadíos larvales. Para los adultos se realizó un análisis de variancia, bajo el diseño estadístico completamente randomizado.

\section{RESULTADOS Y DISCUSIÓN}

\section{Ciclo de Desarrollo}

1. Los huevos son de color blanco cremoso, similar a lo señalado por García (1984). Son elípticos, cuya longitud y ancho son de 0,769 y 0,546, respectivamente. El diseño que presenta es similar a la imbricación de las escamas de un pez. Son puestos en las depresiones de las hojas y en forma aislada o en pequeños grupos de 4 huevos. La mayor cantidad de huevos eclosionan entre el sexto y séptimo día, pero el promedio para los procedentes de los 3 tratamientos es de 7,42 días. El rango de variación de éste período está entre 5 y 10 días. Sin embargo Rázuri y Vargas (1975) determinaron que para Tuta absoluta el período de incubación es de sólo 4 días.

2. La larva, al emerger tiene $1,4 \mathrm{~mm}$ de longitud, el diámetro transversal de la cápsula cefálica es de 0,191 mm y tiene el cuerpo translucido. Este primer estadío larval dura 3,25 días. El segundo estadío larval presenta el diámetro trasversal 0,298 $\mathrm{mm}$ y tiene una duración de 3,04 días. El tercer estadío larval presenta el diámetro transversal de la cápsula cefálica $0,395 \mathrm{~mm}$ y con una longevidad de 2,94 días. Entre el mesotórax y metatórax se observa una zona blanquecina. En éste estado de desarrollo empieza a construir cartuchos. En el cuarto estadío larval el diámetro transversal de la cápsula cefálica es de 0,598 mm y dura 2,99 días. En el aspecto 
dorsal presenta dos líneas longitudinales de color plomizo. El quinto y último estadío larval dura 3,86 días y el diámetro transversal cefálico mide $0,908 \mathrm{~mm}$; y las líneas longitudinales son más oscuras. La caracterización que efectuó Wille (1952) concuerda sólo con el quinto estadío. La prepupa dura 2,79 días. Esta se ubica dentro de un cartucho que fue construida antes de entrar en ésta fase. Rázuri y Vargas (1975) determinaron que las larvas de Tuta absoluta necesitan de 8,41 días para alcanzar el máximo desarrollo, mientras que Brachmia convolvuli emplea 19,10 días.

3. La pupa requiere de 10,20 días para los machos, antes de alcanzar el siguiente estado de desarrollo; y de 9,68 días para las hembras. Presentan 1,310 mg de peso, 7,325 de longitud y 2,035 de diámetro transversal máximo. La cubierta pupal es marrón oscuro y las pterotecas llegan al quinto segmento abdominal y son negruzcas momentos antes de que emerga el adulto. Inicialmente es amarillenta, luego se torna marrón. Se observó en ellas los casos de ojos rojos y ojos negros, de manera similar a lo mencionado por Brits (1980) cuando trabajó con Phthorimaea operculella. El cremaster está formado por 8 ganchitos. La forma consistente para determinar el sexo, es observando la parte ventral de ápice abdominal. Las hembras presentan un mayor distanciamiento entre el poro genital y el poro anal.

4. Los adultos presentan dimorfismo sexual. Las hembras tienen el flagelo microbipectinado a partir del $39^{\circ}$ segmento. El flagelo en ambos sexos tiene 63 segmentos. Los palpos labiales son trisegmentados y curvados; el último segmento es más largo y puntegudo, similar a lo referido por Costa Lima (1945).

\section{Capacidad de Oviposición y Longevidad del Adulto}

Los adultos emergen en las primeras horas del día y después de alimentarse buscan refugio. La actividad empieza en las últimas horas de la tarde y en las noches realizan la cópula, la que es fácilmente interrumpida con el mínimo cambio de intensidad o ruido que se produzca.

El período de pre-oviposición para el primer tratamiento fue de 4,40 días; 5,25 y 5,54 días para el segundo y tercer tratamiento, respectivamente; con un promedio de 5,09 días; pudiendo variar éste período entre 1 y 9 días. Rázuri y Vargas (op. cit.) registraron para Tuta absoluta una variación entre 1 y 4 días, lo que indica que Brachmia convolvuli ovipoista tardíamente. El período de oviposición para los tres tratamientos, respectivamente fue: 21,44; 22,75 y 20,09 días; con un promedio de 21,2 días. El período de post-oviposición, resultó de la siguiente manera: 2,66; 1,75 y 1, 45 días, respectivamente, para cada uno de los tratamientos y el promedio es de 1,92 días.

La longevidad fue de 28,50; 29,75 y 27,08 días, respectivamente, para cada uno de los tratamientos y el promedio fue de 28,29 días. Estos resultados inducen a pensar que cuando la hembra empieza a ovipositar lo antes posible, sufre un desgaste acelerado que cuando empieza más madura (cronológicamente). Una vez cumplida su función, muere.

La fecundidad en el primer tratamiento, la hembra ovipositó un total de 175,70 huevos, con un promedio de 6,16 huevos diarios; mientras que en el segundo tratamiento ovipositó 212 huevos, con un promedio diario de 7, 12 huevos; y en el tercer tratamiento ovipositaron 179, 09 huevos con un promedio diario de 6,61 huevos. El promedio para los tres tratamientos es de 187,40 huevos puestos y un promedio de 6,61 huevos diarios.

Se aprecia una variación de 65 a 367 huevos por hembra, pero Rázuri y Vargas (op. cit.) determinaron para Tuta absoluta una variación entre 100 a 300, lo que indica que Brachmia convolvuli es más variable en cuanto a fecundidad.

La mayor cantidad de huevos son puestos durante los primeros 10 días. Este resultado tiene relación con lo señalado por Brits (1979) debido a que los machos 
probablemente descarguen la mayor cantidad de espermatozoides entre el quinto y décimo día después de la emergencia, puesto que durante este período se obtuvo el mayor número de huevos.

En relación a la fertilidad se señala que al copular los individuos, observados en cada uno de los tres tratamientos, se obtiene 91,63\%; $87,02 \%$ y $87,76 \%$ de huevos fértiles, respectivamente. El promedio es de $88,79 \%$ de huevos fértiles.

Estos resultados indican que cuando los individuos son de la misma edad, existe una mejor sincronización para dar origen a mayor cantidad de huevos fértiles.

\section{CONCLUSIONES}

De los resultados obtenidos en el presente estudio, se concluye:

1. Los huevos son blancos cremosos, elípticos, con una longitud de $0,768 \mathrm{~mm}$ y un ancho de 0,456 mm. El período de incubación dura 7,42 días.

2. Brachmia convolvuli Walsingham presenta cinco estadíos larvales, necesitando un promedio de 18,30 días para su total desarrollo.

3. La duración del estado pupal para las hembras fue de 9,68 días, mientras que para los machos fue de 10,2 días. El peso pupal fue de $1,310 \mathrm{mg}$, el diámetro transversal máximo es de $2,030 \mathrm{~mm}$ y el largo es de 7,320 mm.

4. El período de pre-oviposición tuvo un promedio de 5,09 días. El período de oviposición. de 21,28 días y el de postoviposición, de 1,92 días. La longevidad fue de 28,29 días.

5. La hembra de Brachmia convolvuli ovipuso en promedio 187,70 huevos con un promedio diario de 6,61 huevos. La fertilidad de los huevos varió entre
$96,74 \%$ y $68,75 \%$ con una promedio de $88,8 \%$.

\section{LITERATURA CITADA}

BRITS, J.A. 1979. The influence of age of the adult male reproductive system of the potato tuber moth Phthorimaea operculella . J. Ent.Soc.South Africa 42(2): 395-400.

BRITS, J.A. 1980. The effect of subletal high T on Phthorimaea operculella. J. Ent. South Africa 43(1). 89-106.

CETRARO, L.; M.S. ORTIZ. 1982. Ocurrencia Estacional de Insectos del Camote (Ipomoea batatas, en la Costa Central del Perú. Rev. Per. Ent. 25(1): 17- 32.

COSTA LIMA, A. 1945. Insetos do Brasil. Tomo V. Primera Parte. Escola Nacional de Agronomia, p. 272.

GARCÍA, U. 1984, Apuntes de Laboratorio de Entomología. Mim, UNALM, 1 p.

RÁZURI, V. y E. VARGAS. 1975. Biología y Comportamiento de Scrobipalpula absoluta en tomate. Rev. Per. Ent. 18(1): 84.

SARMIENTO, M. 1983. Las Plagas. Biblioteca Agropecuaria del Perú. P. 67.

WILLE, J. 1952. Entomología Agrícola del Perú. $2^{\circ}$ ed. Revisada y ampliada. Junta de Sanidad Vegetal Ministerio de Agricultura, Lima, p. 409.

\section{AGRADECIMIENTO}

A la Mg. Sc. Clorinda Vergara Cobián, Jefa del Departamento de Entomología y Fitopatología; y Jefa del Museo de Entomología, de la Universidad Nacional Agraria La Molina, por haber facilitado amablemente la actualización de los nombres científicos de Brachmia convolvuli, Tuta absoluta y Microthyris anormalis. 